\title{
Der junge Krebspatient: Psychosoziale Probleme und Konzepte der Rehabilitation
}

\author{
Anna Beraldi ${ }^{\mathrm{a}}$ Sylvia Brathuhn ${ }^{\mathrm{b}} \quad$ Pia Heußner $^{\mathrm{a}} \quad$ Volker König $^{\mathrm{c}} \quad$ Barbara Lawrenz $^{\mathrm{d}}$ \\ aPsycho-Onkologie der Medizinischen Klinik und Poliklinik III, Klinikum der Universität München-Großhadern, \\ ${ }^{b}$ Frauenselbsthilfe nach Krebs e.V., Bonn, \\ 'Klinik für onkologische Rehabilitation und Anschlussheilbehandlung, Bad Oexen, \\ ¿Universitätsfrauenklinik Tübingen, Deutschland
}

\section{Besondere Betreuungsnotwendigkeiten aus der Perspektive der Selbsthilfe \\ Verantwortliche Autorin: Sylvia Brathuhn, Bonn}

Die Diagnose Krebs lässt das Leben aus den Fugen geraten und verändert es auf ungewollte Weise. Die persönliche Konfrontation mit der Krankheit ist für den Betroffenen eine einschneidende Erfahrung, die fast immer als unbarmherzige Zäsur im eigenen Lebensrhythmus erlebt wird [1]. Das trifft auch und gerade auf Krebspatienten zu, die an der Schwelle zum Erwachsenenleben stehen (und also zur Gruppe der «Adolescents and Young Adults» (AYA) gehören). Als AYA werden Patienten recht unterschiedlichen Alters, etwa zwischen dem 16. und 30. Lebensjahr, die zudem eine sehr unterschiedliche Biographie haben, zusammengefasst. Schüler und Auszubildende gehören ebenso in diese Gruppe wie junge Mütter/Väter und Berufseinsteiger. Sie alle brauchen eine individuelle, maßgeschneiderte Behandlung und Betreuung, bei der auf die besonderen Fragen und Ängste junger Menschen mit chronischer Erkrankung eingegangen wird.

Während viele Erwachsene im mittleren und höheren Lebensalter persönliche Schicksalsschläge bzw. «Verluste» bereits kennengelernt haben oder doch gewissermaßen darauf eingestellt sind, sehen sich ältere Jugendliche und junge Erwachsene überwiegend auf der reinen «Gewinnerseite» des Lebens. In ihrer Lebenswaagschale liegen viele positive Erfahrungen (und Erwartungen), und erst in späteren Jahren werden auch negative bzw. belastende Erfahrungen als unvermeidbar hingenommen. Dieser Lernprozess durch allmählichen Wandel wird durch die Diagnose Krebs abrupt verändert. Die Krankheit, die mit erheblichen Verlust- und Einschränkungserfahrungen einhergeht, steht im krassen Gegensatz zum Wesen des späten Jugend- und des jungen Erwachsenenalters, das geprägt ist durch Aufbruch und Aktivität.
Menschen im AYA-Alter wollen sich vom Elternhaus lösen, entsprechend wichtig sind ihnen Werte wie Unabhängigkeit, Selbstbestimmtheit und Anerkennung durch Gleichaltrige. In dieser Phase der Neuorientierung wird eine Krebserkrankung als Stagnation bzw. Rückschritt empfunden, als ein Geschehen, das alte Abhängigkeiten wiederherstellt und zur ungewollten Fremdbestimmung führt. Das geänderte Bindungsbedürfnis der AYA - weg von den Eltern, hin zu Freunden bzw. einem Partner - sollte von Ärzten nicht unterschätzt werden. Junge Menschen identifizieren sich mit Attributen wie aktiv, jung, dynamisch, gesund und lebenshungrig. Die körperliche Unversehrtheit ist in diesen Lebensjahren die tragende und treibende Kraft des Lebens; jedes Sichtbarwerden körperlicher und psychischer Schwächen (durch Narben, Haarverlust, Depressionen, Ängste unter anderem) wird als Makel empfunden. Dem Hunger nach Leben entzieht der Krebs die Nahrung, er macht den Körper passiv und schwach. «Ich empfinde mich als anders, als Sonderling, als Störfall», beschrieb uns eine junge Frau mit Hautkrebs ihre Lebenssituation.

Wie gehen junge Krebspatienten mit ihrer Krankheit um? Wer den Boden unter den Füßen verliert, stellt Fragen nach den existenziellen Werten des eigenen Lebens, besonders dann, wenn das Identitätsgefühl altersbedingt noch im Aufbau ist. Wer bin ich, was sind meine Wurzeln, wo ist mein Platz, wer trägt mich? Diese Sinnsuche wird von den zutiefst verunsicherten Patienten oft durch auffällige Coolness überspielt, eine Strategie, die ja auch von gesunden jungen Menschen in der Phase der Persönlichkeitsfindung angewendet wird. Die Krebserkrankung verstärkt die Identitätsunsicherheit, soeben gefundene oder als erstrebenswert erachtete Lebensziele müssen wieder überdacht werden, neue Fragen drängen sich auf. Die Situation stellt sich von nun an so dar: Der bisher als fast unendlich lang erscheinenden Zukunft mit ihren unzähligen verlockenden Möglichkeiten sind auf einmal

\section{KARGER}

Fax +49761 4520714

Information@Karger.de

www.karger.com (c) 2011 S. Karger GmbH, Freiburg

0378-584X/11/3417-0017\$38.00/0

Accessible online at:

www.karger.com/onk 

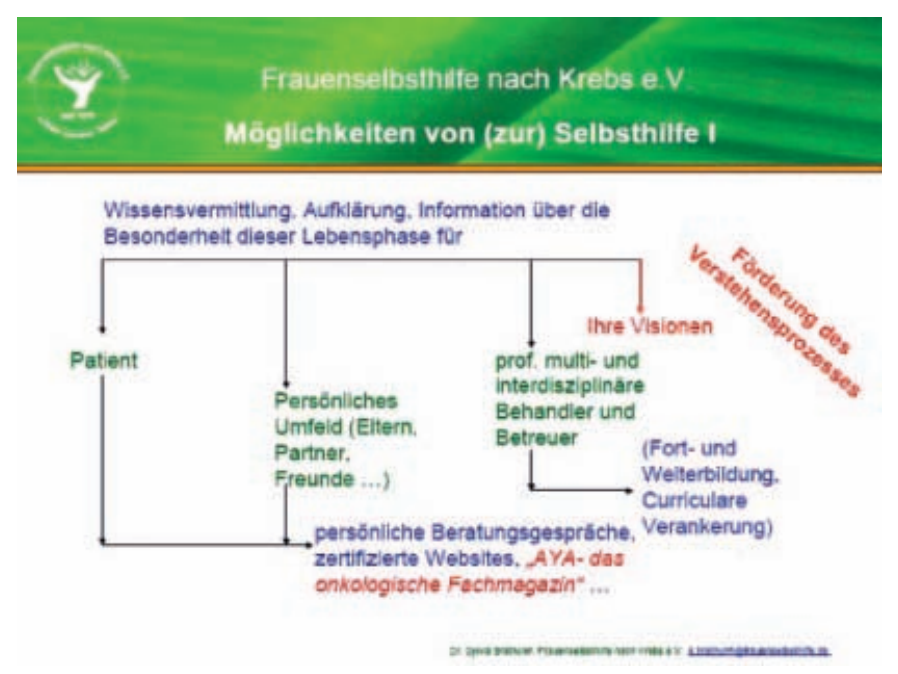

Abb. 1. Möglichkeiten von und zur Selbsthilfe.

enge Grenzen gesetzt, der Lebensplan zerplatzt und an die Stelle von Visionen treten Ängste. Der junge Patient nimmt eine Neubilanzierung seines Lebens vor, indem von nun an die Verlustseite ungleich stärker gewichtet ist als die Gewinnseite. Die Erkenntnis, dass die bisher angewandten Strategien zur Lebensbewältigung nicht mehr greifen, ruft bei vielen Patienten Unverständnis und Verärgerung und damit einhergehend auch oftmals für Ärzte kaum nachvollziehbare Reaktionen hervor. So wird beispielsweise das Verhalten eines jungen Patienten mit niedriger Leukozytenzahl, der trotz aller Warnungen und Verhaltensanweisungen in die Disco geht und sich somit einer großen Gefahr aussetzt, oftmals als persönlicher Affront verstanden. Die Krebserkrankung als Bruch im Lebensplan und ihre daraus resultierenden - nicht immer verständlichen - Reaktionen zu verstehen und zu akzeptieren, ist jedoch ein notwendiger Schritt für alle Beteiligten.

Wie kann dem jungen Krebspatienten geholfen werden? Es gehört zu den ärztlichen Aufgaben, den Patienten die Notwendigkeit und die Erfolgserfolgsausichten onkologischer Therapien zu vermitteln. Dennoch werden die Maßnahmen von Patienten nicht selten als aggressive Eingriffe in ihre Persönlichkeit erlebt, die mit Ausgegrenztsein und Fremdbestimmtheit verbunden sind. Eine Behandlung impliziert jedoch das Wort «handeln», und dies sollte nicht einseitig als ärztliches Handeln verstanden werden. Die aktive Einbindung der Patienten in Therapiebelange kann ihnen das Gefühl der Ohnmacht nehmen und dadurch den Prozess des Verstehens fördern.

Wissensvermittlung und Information der Patienten und ihrer Angehörigen sind in der Betreuung von AYA besonders wichtig, denn sie tragen zur Vertrauensbildung bei. Es könnten verschiedene Angebote gemacht werden, die verstärkt auf die Besonderheiten in der AYA-Lebensphase eingehen (Abb. 1 und 2). Ob sich junge Krebspatienten zu eigenen AYA-Selbsthilfegruppen zusammenfinden werden, sei wegen der geringen Zahl der Betroffenen dahingestellt. Hier bietet

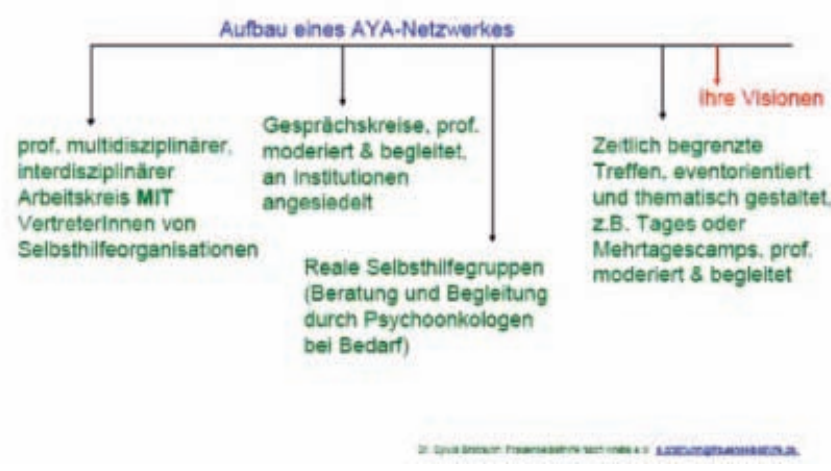

Abb. 2. Möglichkeiten von und zur Selbsthilfe.

das Internet jedoch neue Möglichkeiten, auch für den Aufbau eines moderierten Netzwerkes.

Die Diagnose Krebs fordert von Betroffenen, medizinische Entscheidungen von großer Tragweite zu treffen. Der Patient braucht umfassende Informationen, um sich am Therapiefortgang aktiv beteiligen zu können. Der Erfahrungsaustausch in der Gruppe hilft, den Dschungel an Informationen zu entwirren, individuelle Entscheidungen zu treffen und so einen ganz persönlichen Weg zu finden. Dies betrifft medizinische Entscheidungen, aber es betrifft auch die ganz persönlichen Entscheidungen zur Lebensführung. Denn eine Krebserkrankung ist - unabhängig von Alter, Geschlecht und Profession - eine Zäsur, die das Leben des Betroffenen in ein Davor und Danach zerschneidet. Das Leben muss neu geordnet werden, und darin liegt eine Chance. Im Sog des Alltags, mit beruflichen und familiären Verpflichtungen, vergessen wir uns oft selbst. Der Krebs stoppt unser bisheriges Leben und die unbewusste Illusion, «es werde immer so weitergehen». Er lässt uns innehalten und unser Leben neu sortieren, ruft dazu auf, uns unter Umständen von Belastendem zu trennen und ein Leben im Einklang mit uns selbst zu suchen und bestenfalls zu finden [2].

\section{Psychoonkologische und soziale Probleme bei jungen Patienten mit Leukämie \\ Verantwortliche Autorinnen: Pia Heußner, München, Anna Beraldi, München}

Krebspatienten im jugendlichen und jungen Erwachsenenalter stehen zunächst einmal vor ähnlichen Herausforderungen wie körperlich gesunde junge Menschen - dazu gehören unter anderem die Identitätsfindung und die soziale und berufliche Orientierung (Abb. 3) [3].

Auch wenn das soziale Umfeld eines an Krebs erkrankten jungen Menschen zunächst viel Verständnis signalisiert, blei- 
ben Konflikte bei längerer Berufsunfähigkeit des Patienten bzw. längerer Abwesenheit in Schule, Ausbildungsbetrieb oder Verein nicht aus. Es kann dann ein Berufswechsel anstehen, das Wiederholen eines Schuljahres oder die Suche nach einem neuen, körperlich weniger anstrengendem Hobby. Das alles belastet den Patienten zusätzlich. Im Zusammenhang mit der Therapiesituation - etwa einem längeren stationären Aufenthalt - können sich weitere Problemfelder etwa in den Bereichen Sexualität/Partnerbeziehung und Kontakte zum Freundeskreis (Peergroup) auftun. Krebserkrankung und -therapie stehen dem ausgeprägten Bedürfnis junger Patienten nach Autonomie, Abenteuer und selbstbestimmter Grenzerfahrung entgegen; hier kann auch ein Grund für mangelnde Therapieadhärenz liegen. Hormonelle, physische und kognitive Veränderungen begleiten die Adoleszenz und äußern sich individuell sehr unterschiedlich, müssen aber als Besonderheit in dieser Altersgruppe beachtet werden. Der Einfluss des Lebensalters auf die psychosoziale Belastung ist nicht eindeutig geklärt [4]. Einerseits kann die Gesamtbelastung im höheren Alter ansteigen, andererseits wurde bei jungen Krebspatienten ein höheres $\mathrm{Maß}$ an psychosozialen Belastungen gefunden, auch litten sie stärker unter bestimmten Symptomen und hatten häufiger finanzielle Probleme.

Der Wunsch nach psychosozialer Betreuung wird von Frauen häufiger geäußert als von Männern und von jungen Patienten mehr als von älteren Patienten [5]. Bei Frauen korreliert der Betreuungswunsch mit jüngerem Alter, der Notwendigkeit einer Hormontherapie, einer positiven Einstellung gegenüber Hilfsangeboten (Coping-Strategie) und dem Schweregrad sexueller Probleme. Bei Männern ist der Betreuungswunsch assoziiert mit jüngerem Alter, Berufstätigkeit, der genannten Coping-Strategie und der Schmerzstärke. Bei der positiven Einstellung der AYA-Patientengruppe gegenüber einer psychotherapeutischen Begleitung könnte es sich um einen Kohorten-Effekt handeln, da Menschen in dieser Altersgruppe psychosoziale Hilfsangebote prinzipiell weniger skeptisch beurteilen als ältere Menschen. Auch stehen in der AYA-Lebensphase drängende Probleme in den Bereichen Ausbildung, Arbeitsplatz, Kinder und Partnerbeziehung und finanzielle Sicherung im Vordergrund, weshalb Hilfe gesucht und auch angenommen wird.

Auch der persönliche Umgang mit der Krebserkrankung wird offenbar vom Lebensalter beeinflusst. 6 von 8 Studien zu dieser Thematik kamen zu dem Ergebnis, dass jüngere Patienten (Gruppe der AYA) ihre Krebsdiagnose seltener verleugnen und entsprechend offener darüber reden als ältere Patienten [6]. In 2 Studien korrelierte das Alter nicht mit einem Verleugnen, allerdings zeigten jüngere Patienten mehr «Vermeidungs- und Fluchtverhalten».

Etwa ein Drittel der Patienten mit Krebserkrankungen erhält psychotherapeutische Hilfe [7], so unter anderem das Ergebnis einer kanadischen retrospektiven Datenanalyse von 326 Patienten mit soliden Tumoren. Trotz ähnlicher Depressions- und psychosozialer Belastungswerte wurden die Betreuungswünsche junger Patienten durchweg erfüllt, die der älteren Patienten jedoch nur zu 22\%. Etwa ein Viertel der Patienten war depressiv, doch wurden von ihnen weniger als die Hälfte an einen Psychoonkologen überwiesen. Die Gründe für das ungleiche Überweisungsverhalten sind unklar, womöglich wird der Therapiebedarf jüngerer Patienten höher eingeschätzt bzw. der Bedarf älterer Patienten unterschätzt.

Bei AYA-Patienten gibt es eine Reihe von krankheitsbedingten Herausforderungen, die in der psychoonkologischen Betreuung angesprochen werden sollten. Für viele Patienten ist die «Normalität» des Alltags in weiter Ferne und sie fühlen sich von der neuen Situation überrollt. Der Verlust an Unabhängigkeit, die mögliche soziale Isolation und Veränderungen in Aussehen und Körperbild werden als bedrohlich und unfair erlebt. Unterstützung brauchen Patienten aber auch in Fragen der Compliance, denn es überfordert oft die Motivation, eine «lange» Therapie von mehreren Monaten oder Jahren durchzuhalten. Die selbstgewünschte Eigenverantwortlichkeit des
Abb. 3. Der junge Tumorpatient im sozialen Kontext (modifiziert nach [3]).

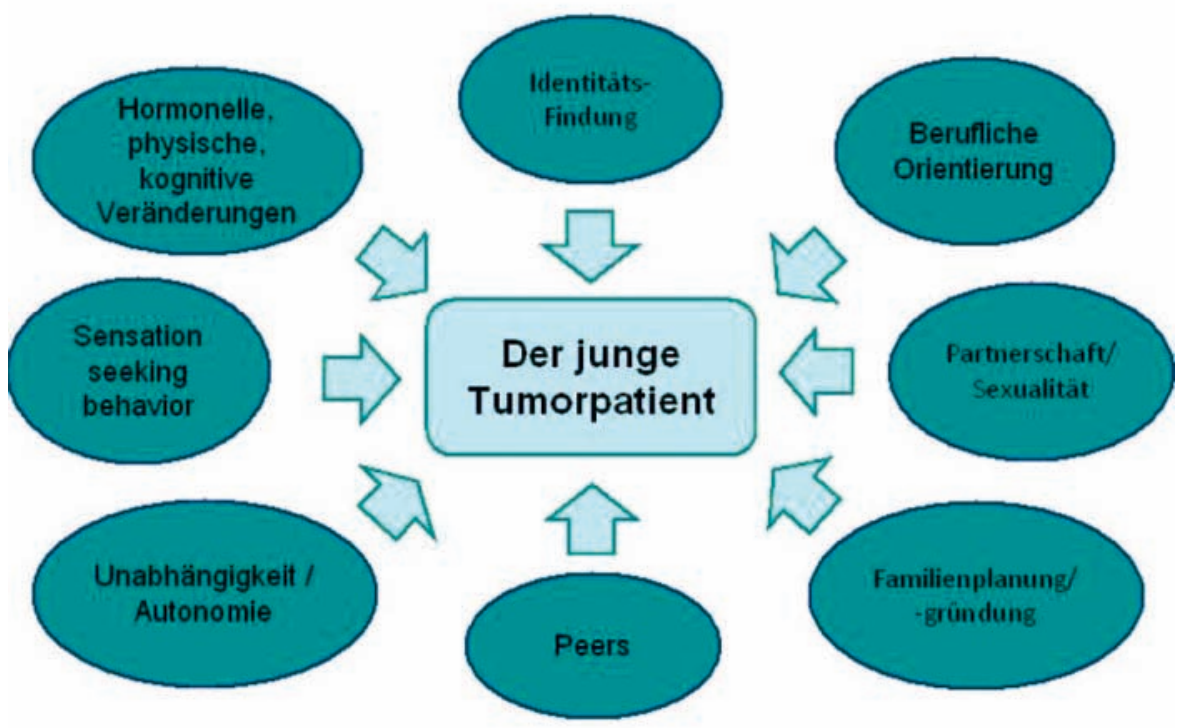


Tab. 1. AYA und Psychotherapie: Was hilft, was ist fehl am Platz? (modifiziert nach [8])

\begin{tabular}{l} 
Hilfreich \\
Klare Information \\
Gleiche Sprache \\
Urteilsfreie Kommunikation \\
Vertrauen \\
Adäquate Umgebung \\
So normal als möglich \\
Adäquate Entwicklung fördernd \\
\hline Nicht hilfreich \\
Paternalistisches Beziehungsmodell \\
Überprotektives oder überkontrollierendes Verhalten \\
Schematische Behandlung als Kind oder reifer Erwachsener \\
\hline
\end{tabular}

Patienten und die notwendige Therapieadhärenz können durch konsequente Anwendung der Grundsätze der partizipativen Entscheidungsfindung (shared decision making) betont und unterstützt werden. Allerdings ist darauf zu achten, dass die geteilte Verantwortung nicht im Rahmen der psychosozialen Belastung zur Überforderung und weiteren Regression führt.

In der psychosozialen Betreuung junger Krebspatienten haben sich einige Strategien als hilfreich erwiesen, während andere den Autonomiebestrebungen dieser Altersgruppe entgegenstehen und deshalb vermieden werden sollten [8]. Hilfreiche Arzt-Patienten-Gespräche setzen unter anderem die Weitergabe klarer Informationen voraus und fördern ein stabiles Vertrauensverhältnis (Tab. 1). Heranwachsende Patienten in der akuten Behandlungssituation benötigen eine andere Begleitung als in der Nachsorge oder als Langzeitüberlebende. Und auch in der palliativen Behandlungsphase ist eine individuell altersadaptierte spezifische psychosoziale Betreuung notwendig.

\section{Fazit}

Aus der komplexen Lebenssituation von Krebspatienten im jugendlichen und jungen Erwachsenenalter ergibt sich ein hoher Bedarf an psychosozialer Unterstützung. Da sich die Fragen und Sorgen junger Patienten von denen älterer Patienten unterscheiden, benötigen AYA eine altersgerechte, systematische Betreuung, die unabhängig von der tatsächlich geklagten Belastung stets angeboten werden sollte. Klare Informationen in verständlicher Sprache werden als hilfreich empfunden. Ein paternalistisches Beziehungsmodell mit überprotektivem oder überkontrollierendem Verhalten wird von den meisten AYA-Patienten abgelehnt - sie wünschen sich vielmehr größtmögliche Autonomie, auch in lebenskritischen Situationen. Um hier den richtigen Weg zu finden, ist eine interdisziplinäre multiprofessionelle Betreuung der Patienten unter Einbeziehung psychoonkologisch geschulter Fachkräfte sinnvoll.

\section{Fertilitätserhalt bei Patientinnen mit Lymphom}

Verantwortliche Autorin: Barbara Lawrenz, Tübingen

Für junge Patientinnen mit Malignomen gibt es heute effektive Chemotherapiekonzepte, die die Prognose deutlich verbessern. Die Chance auf Langzeitüberleben wirft Fragen zur Familienplanung auf, denn $75 \%$ der Frauen wünschen sich nach erfolgreicher Therapie ein Kind [9]. Zugleich prägt die Angst vor einem Rezidiv und den Langzeitfolgen von Krankheit und Therapie das weitere Leben.

Therapien mit gonadotoxischem Potenzial führen zu einer Atresie der Follikel und bergen das Risiko einer Ovarialinsuffizienz, dies ist abhängig von der Zahl der im Ovar vorhandenen Follikel und somit vom Lebensalter, von den verwendeten zytotoxischen Substanzen und einer eventuell notwendigen Radiatio im Bereich des kleinen Beckens. Um die Fertilität nach Chemotherapie zu erhalten, gibt es mehrere Möglichkeiten zur Ovarprotektion, bei deren Auswahl verschiedene Kriterien zu berücksichtigen sind: die Art der onkologischen Therapie (Chemotherapie, Radiatio), die verbleibende Zeit bis zum ersten Chemotherapiezyklus, das Risiko einer ovariellen Metastasierung, der Partnerstatus, das Lebensalter, die Einwilligungsfähigkeit und Wunschvorstellung der Patientin sowie die Kosten des Eingriffes. Da sich die einzelnen Verfahren bezüglich Nebenwirkungen und Vorbereitungszeit unterscheiden, ist die Wahl des jeweils optimalen Verfahrens individuell und sorgfältig zu treffen. Die Optionen stellen sich wie folgt dar.

\section{Kryokonservierung von befruchteten oder unbefruchteten Eizellen}

Bei diesem Verfahren wird zunächst die Eizellreifung hormonell stimuliert, um anschließend mittels vaginaler Follikelpunktion befruchtungsfähige Oozyten zu gewinnen. Die Stimulation kann auch unabhängig vom Menstruationszyklus erfolgen, der Zeitbedarf beträgt etwa 14 Tage. Bei Frauen mit Lebenspartner werden die entnommenen Eizellen in vitro befruchtet - in der Regel wird dafür die intracytoplasmatische Spermieninjektion (ICSI) angewendet - und danach kryokonserviert. Bei Frauen ohne festen Partner werden die nicht befruchteten Eizellen eingefroren. Die Wahrscheinlichkeit einer Schwangerschaft nach Implantation einer kryokonservierten, befruchteten Eizelle beträgt 18-20\%, aus der Verwendung kryokonservierter, nicht fertilisierter Eizellen und nachfolgender ICSI liegt die Chance für eine Schwangerschaft bei etwa $4-5 \%$ pro Eizelle. Bei jungen Patientinnen besteht das Risiko für die Entwicklung eines hormonellen Überstimulationssyndroms, durch die sich der Beginn der Chemotherapie verzögern kann. Eine solche Komplikation ist aber selten; so kam es bei 205 Patientinnen, die eine hormonelle Stimulation vor Chemotherapie erhalten hatten und im FertiPROTEKT-Register erfasst wurden, bei keiner der Frauen zu einer Überstimulation [10]. 


\section{Transposition der Ovarien}

Bei Patientinnen, die aufgrund ihrer Krebserkrankung eine Radiatio im Bereich des kleinen Beckens benötigen, ist von einer Schädigung der Ovarien in Abhängigkeit von der Strahlendosis auszugehen. Bei einer ovariellen Strahlendosis von 2,5-5 Gy beträgt das Risiko für den Verlust der Fertilität etwa $60 \%$. Zum Schutz des Gewebes kann eine ovarielle Transposition vorgenommen werden, was die Strahlenbelastung der Ovarien erheblich senkt. Der Eingriff wird wenn möglich laparoskopisch durchgeführt, dabei werden die Ovarien kraniolateral Richtung Zwerchfell verlagert. Mitunter ist eine Durchtrennung der Eileiter erforderlich; für eine spätere Schwangerschaft ist dann eine In-vitro-Fertilisation nötig.

\section{Kryokonservierung von Ovarialgewebe}

Für die Entnahme und Kryokonservierung von Ovargewebe müssen lediglich 2 Tage reserviert werden, deshalb kann diese Technik auch noch kurzfristig vor einer zytotoxischen Therapie eingesetzt werden. Dafür wird laparoskopisch ein Teil des Ovarkortex eines Ovars reseziert und bis zur Retransplantation (auf das erhaltene Ovar oder in eine Peritonealtasche) kryokonserviert. Die Erfahrungen mit dieser Technik sind sehr begrenzt, bisher wurde von 9 Schwangerschaften nach der Retransplantation von Ovargewebe berichtet. Die Kryokonservierung von Ovarialgewebe ist noch keine Standardtechnik, sie dürfte aber in den nächsten Jahren vor allem jungen Patientinnen - insbesondere solchen, die noch keinen Lebenspartner haben - vermehrt als Alternative zur Kryokonservierung von unbefruchteten Eizellen angeboten werden. $\mathrm{Zu}$ bedenken ist jedoch, dass bei der Retransplantation des Gewebes theoretisch maligne Zellen mit übertragen werden können. Um dieser Frage nachzugehen, wurde kryokonserviertes und wieder aufgetautes Gewebe von 18 LymphomPatientinnen auf immundefiziente Mäuse transplantiert; keines der Tiere entwickelte im späteren Lebensverlauf (Beobachtungszeitraum von 16 Wochen) ein Lymphom [11]. Demnach scheint bei Lymphom-Patientinnen eine Ovargewebsentnahme mit der Option der späteren Retransplantation das Risiko für Rezidivtumore nicht zu erhöhen. Auch andere Komplikationen in Zusammenhang mit der Ovargewebsentnahme sind selten: Von 500 über FertiPROTEKT zur laparoskopischen Ovargewebsentnahme gemeldeten Frauen gab es nur in 2 Fällen $(0,4 \%)$ Komplikationen, einmal konnte der Eingriff wegen Gewebsadhäsionen nicht vorgenommen werden und einmal war ein Wechsel zur Laparotomie wegen Blutungen nötig [12].

\section{Ovarprotektion mit GnRH-Analoga}

Gonadotropin-Releasing Hormone (GnRH)-Agonisten erzeugen innerhalb von etwa 7 Tagen eine Downregulation des GnRH-Rezeptors, gefolgt von einem Hypogonadismus, aufgrund dessen die Ovarien besser vor der Chemotherapie geschützt sein sollen. Die Präparate können erst nach der
Pubertät angewendet werden; sie scheinen weder die Grunderkrankung negativ zu beeinflussen noch die Effektivität der Chemotherapie zu beeinträchtigen.

Ein abschließendes Urteil zur Wirksamkeit der GnRHAnaloga ist noch nicht möglich. Zwar gibt es eine Studie [13] sowie eine Metaanalyse [14], die protektive Effekte nachweisen, aber es liegen auch gegenteilige Ergebnisse vor [15].

\section{Das Netzwerk FertiPROTEKT}

Das Netzwerk FertiPROTEKT wurde 2006 in Heidelberg gegründet. Ziel ist eine flächendeckende Implementierung von Zentren, die kompetent in ganz Deutschland fertilitätserhaltende Maßnahmen anbieten und Krebspatientinnen entsprechend beraten. Über deutsch- und englischsprachige Homepages für Ärzte und Patientinnen (www.fertiprotekt.de) sind alle Zentren des Netzwerks miteinander verlinkt. Es liegen mittlerweile auch Handlungsempfehlungen zur Ovarprotektion vor, ausgehend von den Studien zur Ovartoxizität von Zytostatika im Rahmen von Lymphom-Therapien [16]. In Abhängigkeit vom gewählten Chemotherapie-Regime kommen verschiedene Maßnahmen einzeln oder in Kombination infrage (Abb. 4)

Im Rahmen des Netzwerkes FertiPROTEKT wurden in den Jahren 2007-2009 insgesamt 1280 Patientinnen im Alter zwischen 15 und 40 Jahren beraten [12]. 1080 Patientinnen (84\%) entschieden sich für mindestens eine fertilitätserhaltende Maßnahme. Die vorherrschenden Tumordiagnosen waren Mammakarzinom (34,8\%) und Lymphome (30,5\%; bei den 15 - bis 30 -jährigen Frauen $87,5 \%$ !). Insbesondere bei den Lymphom-Patientinnen stehen die medikamentösen Therapieoptionen zum Ovarschutz im Vordergrund, gefolgt von der Kryokonservierung von Ovargewebe. Wichtig ist in diesem Zusammenhang die frühzeitige Beratung (innerhalb von 1-2 Tagen nach Diagnosestellung) über die Möglichkeiten zum Fertilitätserhalt.

In Tübingen wurden zwischen 11/2006 und 01/2010 insgesamt 111 Lymphom-Patientinnen hämato-onkologisch betreut, davon waren 39 Patientinnen unter 40 Jahre alt. Von

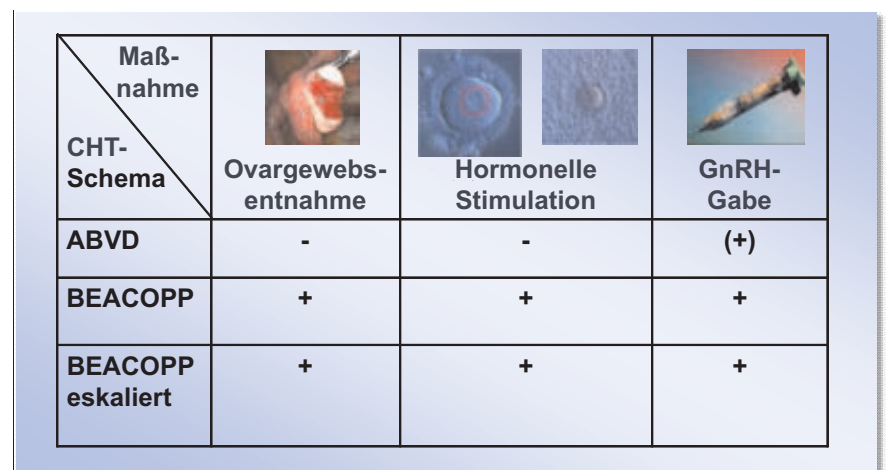

Abb. 4. Ovarprotektive Maßnahmen für Lymphom-Patientinnen (aus [18], mit freundlicher Genehmigung, Copyright Krause \& Pachernegg GmbH, Gablitz) 
den 27 Patienten aus der AYA-Altersgruppe (Durchschnittsalter 25,8 Jahre) entschieden sich $96 \%$ für die GnRH-Gabe, bei $27 \%$ wurde zusätzlich Ovargewebe entnommen und bei $23 \%$ eine hormonelle Stimulation zur Gewinnung und Kryokonservierung der zuvor befruchteten Eizellen vorgenommen. Bei einer Patientin wurden alle 3 Maßnahmen kombiniert. Der Zeitbedarf für Laparoskopie und Ovargewebsentnahme betrug median 2 Tage, nach median weiteren 6 Tagen konnte die Chemotherapie beginnen. Für die hormonelle Stimulation zur Oozytengewinnung waren median 17,5 Tage nötig, die Chemotherapie begann dann nach weiteren 4,5 Tagen [17].

\section{Fazit}

Fertilitätsprotektive Maßnahmen können gut in das onkologische Therapieregime integriert werden, dafür ist jedoch eine enge Kooperation zwischen hämato-onkologischen Abteilungen und reproduktionsmedizinischen Zentren notwendig. Eine frühzeitige Beratung ist wünschenswert, um in Abhängigkeit von der onkologischen Therapie die am besten geeignete Maßnahme zum Fertilitätserhalt wählen zu können.

\section{Besondere Aufgaben der Rehabilitation \\ Verantwortlicher Autor: Volker König, Bad Oexen}

Die stationäre Rehabilitation junger Erwachsener mit malignen Erkrankungen hat zum Ziel, durch Steigerung der körperlichen Leistungsfähigkeit und Linderung von tumorund therapiebedingten Störungen die Patienten somatisch, psychisch, beruflich und sozial zu stabilisieren. Eine chronische Pflegebedürftigkeit sollte wenn irgend möglich verhindert werden. Die Gruppe, für die unsere «Junge Erwachsenen Reha» (JER) als spezifisches Angebot entwickelt wurde, betrifft Patienten mit Krebserkrankung im Alter von 18-32 Jahren (AYA-Zielgruppe). Häufigste Diagnosen bei den Patienten $(\mathrm{n}=923)$, die seit 2001 eine Junge-Erwachsenen-Rehabilitation in der Klinik Bad Oexen erhalten haben, waren Lymphome und Leukämien (45\%), urologische Tumoren (22\%, überwiegend Hodentumoren) und gynäkologische Tumoren (13\%, davon 6\% Mammakarzinome und 4\% Ovarialkarzinome).

Die Gruppe der AYA unterscheidet sich in einigen Aspekten von den Erwachsenen: So sind die Persönlichkeitsentwicklung und die Partnerwahl bei ihnen in der Regel noch nicht abgeschlossen, der Ablöseprozess von den Eltern ist krankheitsbedingt verzögert und oft muss die Berufsausbildung unterbrochen werden. All dies bringt die jungen Menschen in multiple Krisensituationen, was im Rehabilitationskonzept berücksichtigt werden muss. Im regulären RehaBetrieb ist dies kaum möglich, da für die zahlenmäßig kleine Gruppe der AYA weder für deren Alter ansprechende Räumlichkeiten noch spezielle psychologische Unterstützungskonzepte vorhanden sind. Auch auf das erhöhte Infor-
Tab. 2. Vorstellungen junger Erwachsener mit malignen Erkrankungen an eine Rehabilitation

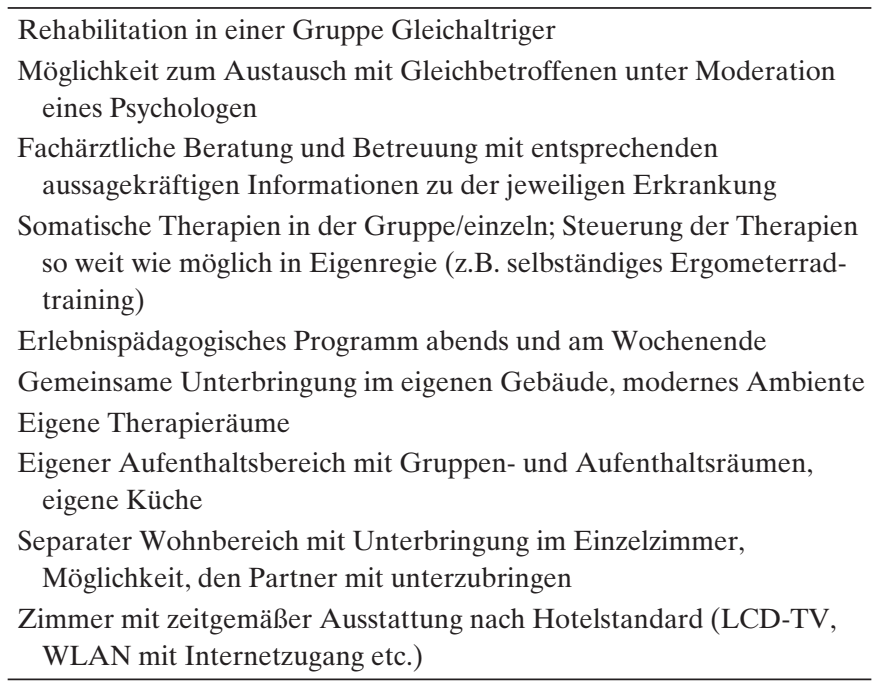

mationsbedürfnis und die Fragen zur Berufsfindung seitens der jungen Patienten sind allgemeine Reha-Einrichtungen nicht ausreichend eingestellt. Vielmehr muss das Angebot auf die Vorstellungen und Wünsche der AYA (Tab. 2) zugeschnitten sein, wie dies etwa in der Klinik Bad Oexen mit dem speziellen Programm JER seit 1987 umgesetzt wird (www.jer-reha.de).

Der für den Rehabilitationserfolg so wichtige Austausch zwischen Betroffenen gleichen Alters gelingt am besten in Gruppen mit überschaubarer Größe; in der Bad Oexener Klinik bilden bei einer Gesamtkapazität von 48 Therapieplätzen jeweils 12 Rehabilitanden eine eigene Gruppe, die in fester Zusammensetzung für 4 Wochen zusammenbleibt. Inhaltlich setzen wir auf eine aktivierende Rehabilitation mit dem Schwerpunkt der Kompetenzvermittlung und Stärkung der Selbsthilfefähigkeit. Das Angebot umfasst

- Sport-/Physio-/Bewegungstherapie

- psychologische Betreuung im Gruppensetting

- Gesundheitsinformation und -motivation

- sozialrechtliche Beratung/Berufsberatung

- Ernährungstherapie/-beratung

- Erlebnispädagogik.

Krankheits- und therapiebedingt kann eine Reihe von Funktionseinschränkungen auftreten, deren Behandlung im Rahmen der JER-Rehabilitation gezielt angegangen wird. Art und Ausmaß der Funktionsstörungen werden durch die Primärtherapie bestimmt, im Wesentlichen handelt es sich um Organdysfunktionen (etwa als Folge von Bestrahlung oder Chemotherapie), um Störungen des Körperbildes und vermehrte psychosoziale Belastungen. Die häufigsten therapieassoziierten Funktionsstörungen sind in Tabelle 3 zusammengefasst.

Vor Beginn des individuell zusammengestellten Reha-Programms erfolgt eine ausführliche Rehabilitationsdiagnostik 
Tab. 3. Funktionsstörungen im Zusammenhang mit Krebstherapien

Rehabilitationskonzept bei jungen Erwachsenen mit maligner Erkrankung

Aktivierende Rehabilitation mit dem Schwerpunkt der Kompetenzvermittlung (Verbesserung der Selbsthilfefähigkeit)

Die Betreuung erfolgt in der geschlossenen Gruppe, d.h. es kommen nach Etablierung der Gruppe keine neuen Rehabilitanden hinzu Sport-/Physio-/Bewegungstherapie

Psychologische Betreuung im Gruppensetting

Gesundheitsinformation und -motivation

Sozialrechtliche Beratung/Berufsberatung

Ernährungstherapie/-beratung

Erlebnispädagogik

Therapieassoziierte Funktionsstörungen (seltener)

Gastrointestinale Beschwerden $\rightarrow$ Übelkeit, Erbrechen, Durchfall, Obstipation, Inappetenz, Gewichtsverlust

Chemotherapie, Operation, Radiatio

Kardiotoxizität $\rightarrow$ Atemnot bei Belastung, Schwäche, Müdigkeit, Schwindel

Anthrazykline, Trastuzumab, 5-FU, Zustand nach Mediastinalbestrahlung

Pneumotoxizität $\rightarrow$ Atemnot bei Belastung

Bleomycin, Etoposid, Cyclophosphamid, Zustand nach Mediastinalbestrahlung

Niereninsuffizienz $\rightarrow$ Ödeme, Störungen der Salz-/Wasserregulation Cisplatin, Alkylantien, Nitrosoharnstoffe, Methotrexat, Mitomycin, Vancomycin, Amphotericin B

Zytopenie (Leuko-, Thrombopenie, Anämie) $\rightarrow$ Infektgefährdung, Blutungsneigung, Schwäche, Atemnot

Chemotherapie, Operation, Radiatio zur Abschätzung von Regulationsbreite und Funktionskapazität der Organsysteme. Dazu gehören eine körperliche Untersuchung, eine Labor-Basisdiagnostik und bei Bedarf eine apparative Funktionsdiagnostik wie die Spirometrie, Bodyplethysmographie und Ergometrie. Bei therapieassoziierten Konzentrations- bzw. Merkfähigkeitsstörungen kann ein Hirnleistungstest durchgeführt werden, auch stehen für die psychologische Basisdiagnostik verschiedene Testinstrumente zur Verfügung. Nach Abschluss der Diagnostik werden die Therapieziele formuliert und ein Therapieplan festgelegt. Eine Besonderheit in der Rehabilitation junger Erwachsener ist sicherlich der Baustein Erlebnispädagogik. Dazu gehören verschiedene Aktivitäten unter pädagogischer Anleitung. Sie haben zum Ziel, das Selbstbewusstsein der Patienten zu stärken nach dem Motto: «Das kann ich wieder.» Angeboten werden unter anderem das Klettern an der Kletterwand und Kanufahren, aber auch Ausflüge, bei denen das «sich unter die Leute wagen» wieder geübt wird.

\section{Disclosure Statement}

Es bestehen keine Interessenkonflikte.

\section{Literatur}

1 Brathuhn S: Diagnose Krebs: Wenn Gedanken keinen Halt mehr finden und Worte zu Flüchtlingen werden. Ärztliche Psychotherapie 2010;5:182-187.

2 Brathuhn S: Selbst ist die Frau. SIGNAL 2007;4.

3 Butow $\mathrm{P}$ et al.: Review of adherence-related issues in adolescents and young adults with cancer. J Clin Oncol 2010;28:4810-4818.

4 Arndt V et al.: Quality of life in patients with colorectal cancer 1 year after diagnosis compared with the general population: a population-based study. J Clin Oncol 2004:22:4829-4836.

5 Merckaert I et al.: Cancer patients' desire for psychological support: prevalence and implications for screening patients' psychological needs. Psychooncology 2010;19:141-149.

6 Vos MS et al.: Denial in cancer patients, an explorative review. Psychooncology 2007;16:12-25.

$\checkmark 7$ Ellis J et al.: Predictors of referral for specialized psychosocial oncology care in patients with metastatic cancer: the contributions of age, distress, and marital status. J Clin Oncol 2009;27:699-705.

8 Morgan S et al.: Sex, drugs, and rock 'n' roll: caring for adolescents and young adults with cancer. J Clin Oncol 2010;28:4820-4830.
Schover LR et al.: Having children after cancer. A pilot survey of survivors' attitudes and experiences. Cancer 1999;86:697-709.

10 Lawrenz B et al.: Efficacy and safety of ovarian stimulation before chemotherapy in 205 cases. Fertil Steril 2010;94:2871-2873.

11 Kim SS et al.: Ovarian tissue harvested from lymphoma patients to preserve fertility may be safe for autotransplantation. Hum Reprod 2001;16:20562060.

12 Lawrenz B et al.: Management of fertility preservation in young breast cancer patients in a large breast cancer centre. Arch Gynecol Obstet 2010; 282:547-551.

13 Badawy A et al.: Gonadotropin-releasing hormone agonists for prevention of chemotherapy-induced ovarian damage: prospective randomized study. Fertil Steril 2009;91:694-697.

14 Blumenfeld Z, von Wolff M: GnRH-analogues and oral contraceptives for fertility preservation in women during chemotherapy. Hum Reprod Update 2008;14:543-552.
5 Behringer $\mathrm{K}$ et al.: No protection of the ovarian follicle pool with the use of GnRH-analogues or oral contraceptives in young women treated with escalated BEACOPP for advanced-stage Hodgkin lymphoma. Final results of a phase II trial from the German Hodgkin Study Group. Ann Oncol 2010;21:2052-2060.

16 Behringer $\mathrm{K}$ et al.: Secondary amenorrhea after Hodgkin's lymphoma is influenced by age at treatment, stage of disease, chemotherapy regimen, and the use of oral contraceptives during therapy: a report from the German Hodgkin's Lymphoma Study Group. J Clin Oncol 2005;23:7555-7564.

17 Lawrenz B et al.: Fertility preservation in young female lymphoma patients: review of available options and initial experiences with their implementation in clinical routine. Onkologie 2011;34:88-93.

18 Lawrenz B et al.: Ferti PROTEKT - Netzwerk für fertilitätsprotektive Maßnahmen bei Chemo- und Strahlentherapie. J Reproduktionsmed Endokrinol 2010;7:147-150. 